\title{
Four butyrolactones and diverse bioactive secondary metabolites from terrestrial Aspergillus flavipes MM2: isolation and structure determination
}

\author{
Mohamed MS Nagia ${ }^{1,3}$, Mohammad Magdy El-Metwally ${ }^{2}$, Mohamed Shaaban ${ }^{1,3^{*}}$, Soheir M El-Zalabani ${ }^{4}$ and
} Atef $G$ Hanna ${ }^{1}$

\begin{abstract}
The chemical constituents and biological activities of the terrestrial Aspergillus flavipes MM2 isolated from Egyptian rice hulls are reported. Seven bioactive compounds were obtained, of which one sterol: ergosterol (1), four butyrolactones: butyrolactone I (2), aspulvinone H (3), butyrolactone-V (6) and 4,4'-diydroxypulvinone (7), along with 6-methylsalicylic acid (4) and the cyclopentenone analogue; terrien (5). Structures of the isolated compounds were deduced by intensive studies of their 1D \& 2D NMR, MS data and comparison with related structures. The strain extract and the isolated compounds (1-7) were biologically studied against number of microbial strains, and brine shrimp for cytotoxicity. In this article, the taxonomical characterization of A. flavipes MM2 along with its upscale fermentation, isolation and structural assignment of the obtained bioactive metabolites, and evaluate their antimicrobial and cytotoxic activities were described.
\end{abstract}

Keywords: Aspergillus flavipes MM2, butyrolactones, biological studies

\section{Background}

In recent years, numerous metabolites possessing uncommon structures and potent bioactivity have been isolated from strains of bacteria and fungi collected from diverse environments, such as soils, animals, plants and sediments [1,2]. It was noted until Alexander Fleming discovered penicillin G from Penicillium notatum almost 83 years ago (1928) that fungal microorganisms suddenly became a hunting ground for novel drug leads $[3,4]$. Therefore, many pharmaceutical companies and research groups were motivated to start sampling and screening large collections of fungal strains for antibiotics $[3,5,6]$. Antimycotics [7,8], antivirals [9], anticancers [10] and pharmacologically active agents [11]. The Aspergilli represents a large diverse genus, containing ca. 180 filamentous fungal species, of substantial

\footnotetext{
* Correspondence: mshaaba_99@yahoo.com

'Division of Pharmaceutical Industries, Chemistry of Natural Compounds Department, National Research Centre, El-Behoos st. 33, Dokki, Cairo 12622, Egypt

Full list of author information is available at the end of the article
}

pharmaceutical and commercial values [12]. In the research program to explore promising bioactive secondary metabolites from fungi, the terrestrial fungi, Aspergillus flavipes sp. isolate MM2 obtained from rice hulls, was investigated. The strain extract revealed the presence of promising antimicrobial activity against some pathogenic test organisms. Chemical screening (TLC investigation) of the strain extract showed numerous characteristic bands. Therefore, the strain was applied to large-scale fermentation by using CzapeckDox medium [13]. Working up of the strain cells produced ergosterol (1), while the filtrate extract afforded six diverse metabolic compounds: butyrolactone-I (2), aspulvinone $\mathrm{H}(3), 6$-methylsalicylic acid (4), terrien (5), butyrolactone-V (6) and 4,4'-diydroxypulvinone (7). The chemical structures of the isolated compounds (1-7) were identified with the help of NMR (1D \& 2D) and mass spectrometry (ESI, EI, HRESIMS) (Figure 1). The antimicrobial activity was tested against some microorganisms and cytotoxicity was examined by using brine shrimp. 


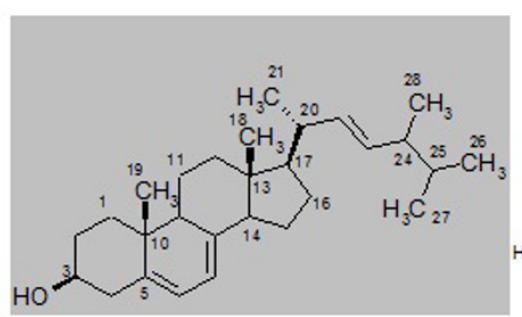

1<smiles>C=C(C)/C=C/C1=C(O)/C(=C/c2ccc(O)c(CC=C(C)C)c2)OC1=O</smiles>

3

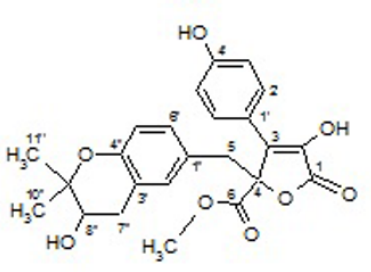

6

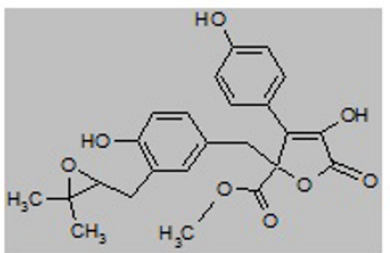

9

4

7

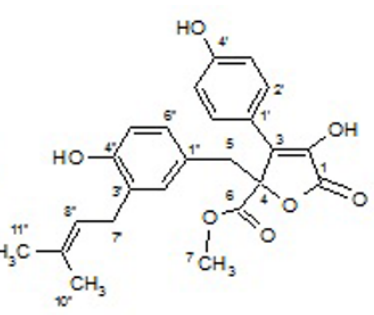

2
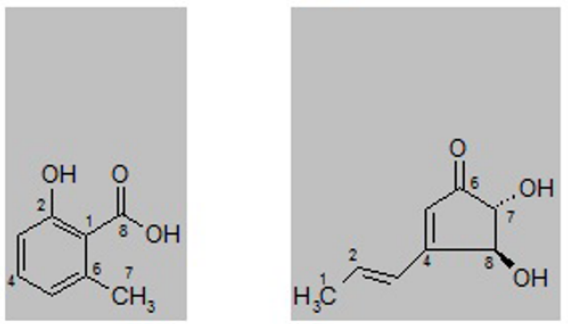

5

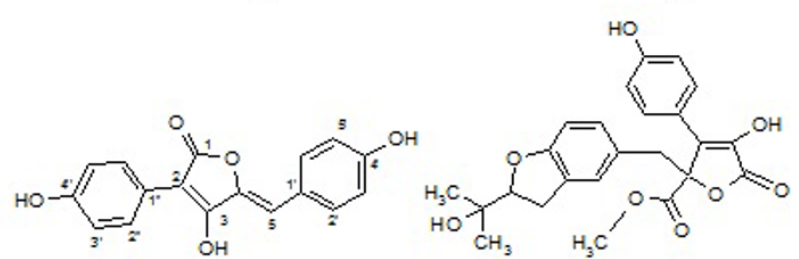

8

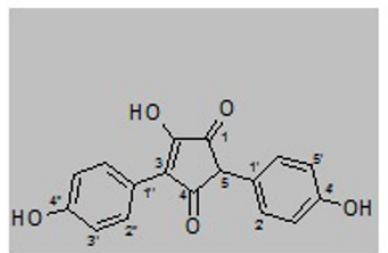

10

Figure 1 Structural formula of the investigated compounds (1-10)

\section{Results and discussion}

\subsection{Taxonomical characterization of the fungal strain}

The grown colonies of the fungal strain on Czapek-Dox medium showed bright whit-faint yellow colonies on the agar plate with a brown staining background [13]. The colonies are growing rather slowly, showing whitish from conidial masses, with brownish conidiophores shining through, reverse yellow-brown to red brown conidial heads spas, loosely columnar, conidiophores smooth-walled, pale yellow to light brown 2.4-3.2 $\mu \mathrm{m}$ in diameter. According to its morphological and microscopic characteristics and comparison with the taxonomical keys of Raper and Fennel [14], the strain was assigned as A. flavipes MM2.

\subsection{Fermentation, working up and isolation}

Based on the pre-screening study, the fungal strain $A$. flavipes MM2, cultivated on Czapeks-Dox for 10 days at $28^{\circ} \mathrm{C}$, was shown to exhibit biological and chemical interest results. Therefore, the fungal strain was scaled up as $10 \mathrm{~L}$ culture using the same cultivating conditions applied for screening studies. After harvesting, both supernatant and mycelial cake phases were individually worked up. Purification of the mycelial extract using silica gel column, followed by washing the afforded major fraction by methanol and purification with Sephadex LH-20, yielded ergosterol (1). An application of the culture filtrate extract of $A$. flavipes MM2 to silica gel column chromatography, followed by diverse 
chromatographic techniques, resulted in the isolation of six compounds: butyrolactone-I (2), aspulvinone $\mathrm{H}(3)$, methylsalicylic acid (4), terrine (5), butyrolactone-V (6) and 4,4'-diydroxypulvinone (7).

\subsection{Chemical characterization}

\subsubsection{Ergosterol (1)}

Ergosterol (1) was obtained as colourless solid, showing UV activity during TLC, which turned violet on spraying with anisaldehyde/sulphuric acid and changed latter to blue. Structure of $\mathbf{1}$ was confirmed by different spectroscopic means (EI MS, $\left.{ }^{1} \mathrm{H},{ }^{13} \mathrm{C} / \mathrm{APT} \mathrm{NMR}\right)$, chromatographic and comparison with literature $[15,16]$. Ergosterol plays an important role as inhibitor of lipid per-oxidation and showed strong DPPH radical scavenging activity as well $[17,18]$, along with its cytotoxicity against HL-60 cells [19], MCF-7 cell line [20].

\subsubsection{Butyrolactone-I (2)}

The molecular weight of 2 was established as 424 Dalton by ESI MS, having the corresponding molecular formula $\mathrm{C}_{24} \mathrm{H}_{24} \mathrm{O}_{7}$ and 13 unsaturation bonds. ${ }^{1} \mathrm{H} / \mathrm{H}, \mathrm{H}$ COSY NMR spectra of 2 showed two o-doublets $(J \sim$ $8.8 \mathrm{~Hz}$ ) each of $2 \mathrm{H}$ at $\delta 7.57$ and 6.86 , being for 1,4-disubstituted aromatic residue, along with three signals at $\delta$ $6.50,6.48$ and 6.40 representing 1,3,4-trisubstituted aromatic ring. A triplet signal of $1 \mathrm{H}$ was at $\delta 5.05$, representing an olefinic methine linked to a doublet methylene signal appeared at $\delta 3.06$. A $3 \mathrm{H}$ methoxy group (3.76); doublet of an $\mathrm{AB}$ methylene group ( $\delta 3.42$ ) attached to $s p^{2}$ system; and further two singlet methyls were visible at $\delta 1.65$ and 1.56 , representing a prenyl system.

According to the ${ }^{13} \mathrm{C}$ NMR/HMQC spectra of compound 2, 22 carbon signals representing 24 carbons were displayed, including 2 carbonyls ( $\delta 171.6$ and $170.3), 2 s p^{2}$ oxygenated carbons $(\delta 159.3,155.0)$ of phenolic systems, along with 5 quaternary carbons $(\delta$ 139.6123.1). Two $2 \mathrm{CH} s p^{2}$ methine signals (130.4 and 116.6) for 1,4-disubstituted benzene ring beside to four $s p^{2}$ methines $(\delta 132.4-115.0)$. In the aliphatic region, signals for quaternary oxygenated methine $(\delta 86.8)$, methoxy (53.8), two methylenes $(\delta 39.6,28.7)$ and two methyls $(\delta$ $25.9,17.8)$ were assigned. Finally, structure of 2 was further deduced on the basis of HMBC experimental data, and comparison with literature as Butyrolactone-I $[21,22]$. Butyrolactone-I (2) was reported as a lipid lowering agent of Lovastatin $\times[19,23,24]$, showing antiproliferative activity against colon, pancreatic carcinoma, human lung cancer and prostatic cancer [25-30] (Figure 2).

\subsubsection{Aspulvinone $H$ (3)}

Based on the ESI MS, the molecular weight of $\mathbf{3}$ was deduced as 432 Dalton, and the corresponding molecular formula as $\mathrm{C}_{27} \mathrm{H}_{28} \mathrm{O}_{5}$, containing 14 unsaturation bonds, as closely related to butyrolactone-I (2). ${ }^{1} \mathrm{H}$ NMR spectrum of 3 showed six confused doublets each of $1 \mathrm{H}$ between $\delta 7.81$ and 6.73 , being of two unsymmetrical tri-substituted aromatic residues, and singlet methine at $\delta 6.22$. A multiplet of $2 \mathrm{H}(\delta 5.36), 4 \mathrm{H}$ of two attached $s p^{2}$-bounded methylenes $(\delta 3.40-3.00)$ and multiplet signal $(\delta 1.75,12 \mathrm{H})$ of four $s p^{2}$-linked methyls, assigning two prenyl systems. Based on the revealed NMR data and molecular formula, and search in AntiBase [2], structure of $\mathbf{3}$ was fixed as aspulvinone $H$ [31].

\subsubsection{6-Methylsalicylic acid (4)}

According to the ESI mass spectra, the molecular weight of 4 was deduced as 152 Dalton. The ${ }^{1} \mathrm{H}$ NMR spectrum displayed three $1 \mathrm{H}$ resonating signals in the aromatic region $(7.07,6.63,6.59)$, being of $1,2,3$ trisubstiuted aromatic ring $(J \sim 7.5-8.2 \mathrm{~Hz})$ together with a singlet $3 \mathrm{H}$ of an aromatic bounded methyl $(\delta$ 2.56). Based on the ${ }^{13} \mathrm{C}$ NMR spectrum, compound 4 displayed eight carbon signals, including one quaternary $(\delta$ 176). Two further deep field quaternaries were visible $(\delta 162.7$ and 142.7$)$ for a-peri-hydroxy and methyl-sp $p^{2}$ attached carbons, respectively. Three $s p^{2}$ methines $(\delta$ $132.2,122.9,115.1)$, one quaternary $(\delta 119.5)$ and a methyl signal ( $\delta$ 23.3). In accordance, 2-Hydroxy-6methyl-benzoic acid (4) was recognized [32,33] as antifungal substance [34], in addition to its analgesic [35], herbicidal [36] and antiacne activities [37].

\subsubsection{Terrein (5)}

The molecular weight of $\mathbf{5}$ was deduced as 154 Dalton $\left(\mathrm{C}_{8} \mathrm{H}_{10} \mathrm{O}_{3}\right)$, bearing four double bond equivalents. The ${ }^{1} \mathrm{H}$ NMR/H,H COSY spectrum of $\mathbf{5}$ showed two signals at $\delta 6.82$ and $6.42(J \sim 15 \mathrm{~Hz})$, representing a trans-olefinic double bond, attached to a doublet methyl ( $\delta$ 1.97), constructing a terminal propene system. A further singlet ( $\delta 5.99)$ being of an olefinic methine and two doublets each of $1 \mathrm{H}(\delta 4.67,4.07, J \sim 2.8 \mathrm{~Hz})$ corresponding to adjacent oxy-methines. According to ${ }^{13} \mathrm{C} \mathrm{NMR} /$ HMQC spectra, eight carbon signals were displayed, including an acetophenone carbonyl $(\delta$ 205.6) and a deep field $s p^{2}$ quaternary carbon $(\delta 170.8)$; three $s p^{2}$ methines ( $\delta 141.8,126.4$ and 125.9$)$, two $s p^{3}$ oxymethines $(\delta 82.4,78.1)$ and one $s p^{2}$-bounded methyl group (19.5).

A final interpretation of $\mathbf{5}$ was carried out by HMBC experiment (Figure 3), fixing the structure as 4,5-dihydroxy-3-propenyl-cyclopent-2-enone; terrein [38,39]. Terrein (5) has a hypopigmentary effect in Mel-Ab cells, and it is a strongly down-regulator of melanin synthesis by reducing tyrosinase production [40], and inhibit human platelet aggregation [41]. Terrein showed a strong antiproliferative effect on skin equivalents [42] and as proteasome inhibitor and anti-tumoral drug [43]. 


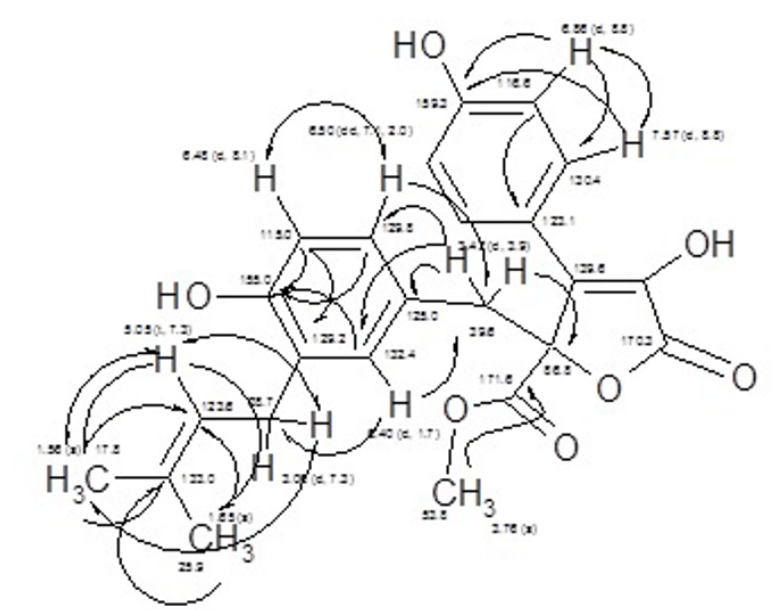

Figure $2 \mathrm{H}, \mathrm{H}$ COSY $(\leftrightarrow)$ and HMBC $(\rightarrow)$ correlations of butyrolactone-I (2).

\subsubsection{Butyrolactone-V (6)}

The molecular weight of 6 was established as 440 Dalton $\left(\mathrm{C}_{24} \mathrm{H}_{24} \mathrm{O}_{8}\right)$, containing 13 double bond unsaturations. The ${ }^{1} \mathrm{H}$ NMR/H,HCOSY spectrum of compound 6 revealed the presence 1,4-disubstituted $(\delta 7.54$ and $6.85, J \sim 8.8 \mathrm{~Hz})$, and unsymmetrical tri-substituted $(\mathrm{m}$, $\delta$ 6.48) aromatic systems. A $1 \mathrm{H}$ dd signal $(\delta 5.02)$ of an oxygenated methine attached to a dd signal $(\delta 2.80)$ of a methylene group, confirming their ABX property. A singlet of methoxy group $(\delta 3.77), 2 \mathrm{H}$ methylene singlet $\left(\delta\right.$ 3.40) flanked by two $s p^{2}$ systems, and two methyl singlets ( $\delta 1.25$ and 1.16), being of gem dimethyl groups were deduced.

Based on the ${ }^{13} \mathrm{C}$ NMR/HMQC spectra of compound 6, two carbonyls ( $\delta 171.5$ and 170.3), two phenolic carbons $(\delta 159.4$ and 153.3), $\beta$-quaternary carbon $(\delta 139.6)$ of an ester or lactone system were deduced. Two $2 \mathrm{CH}$ $s p^{2}$ signals $(\delta 130.4,116.6)$, and $3 \mathrm{CH} s p^{2}$ signals $(\delta$ $132.9,130.4,117.2)$, being of 1,4-disubstitued and

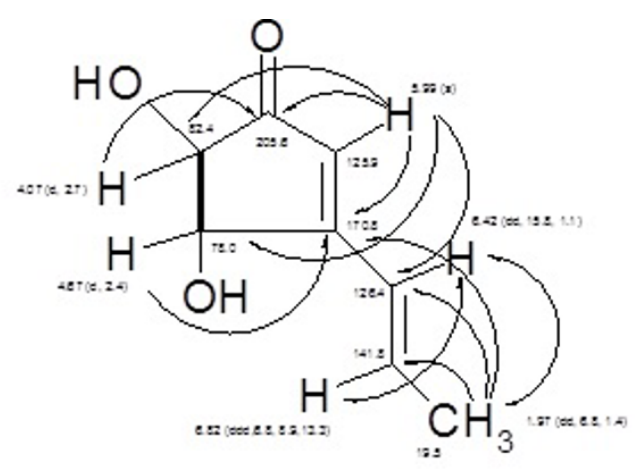

Figure $3 \mathrm{H}, \mathrm{H} \operatorname{COSY}(-)$ and $\mathrm{HMBC}(\rightarrow)$ correlations of Terrein (5). unsymmetric tri-substituted phenolic residues were shown. Two $s p^{3}$ quaternary oxy-carbons $(\delta 86.8$ and $78.0)$, one oxy-methine $(\delta 70.4)$, one carbomethoxy $(\delta$ $53.9)$ and two methylenes $(\delta 39.0,2.80)$ were visible. Two gem dimethyl signals attached to an oxygenated quaternary carbon were displayed $(\delta 25.9,20.9)$.

Based on the above spectroscopic data and molecular formula, compound $\mathbf{6}$ exhibited a strong close structural similarity to butyrolactone-I (2). In accordance, three structural formulas were proposed according to search in AntiBase: butyrolactone-V (6) [44,45], 4-hydroxy-2[2-(1-hydroxy-1-methyl-ethyl)-2,3-dihydro-benzofuran5-ylmethyl]-3-(4-hydroxy-phenyl)-5-oxo-2,5-dihydrofuran-2-carboxylic acid methyl ester (8) [21] and butyrolactone-III (9) [19].

The structure was confirmed by detailed 2D experiments; $\mathrm{H}, \mathrm{H}$ COSY and HMBC (Figure 4) and comparison with literature as butyrolactone- $\mathrm{V}(6)[25,42,43]$. Butyrolactone- $\mathrm{V}$ was reported to exhibit a moderate antimalarial activity against the malarial parasite Plasmodium falciparum K1 (IC50 $7.9 \mu \mathrm{g} / \mathrm{mL}$ ) [45].

\subsubsection{4,4'-Diydroxypulvinone (Aspulvinone E) (7)}

Compound 7 was obtained with a molecular weight of 296 Dalton $\left(\mathrm{C}_{17} \mathrm{H}_{12} \mathrm{O}_{5}\right)$ by HRESI MS, bearing 12 double bond equivalents. The ${ }^{1} \mathrm{H}$ NMR spectrum of 7 showed five signals in the aromatic region for $9 \mathrm{H}(\delta$ 8.06-5.88), representing two 1,4-disubstitued phenolic residues $(J \sim 8.6 \mathrm{~Hz})$. The fifth signal $(1 \mathrm{H})$ was shown as singlet at $\delta 5.88$. Based on the revealed spectroscopic data and molecular formula and search in AntiBase, two alternatives, 4,4'-diydroxypulvinone (7) and Gyrocyanin (10) were displayed. However, the chemical shift of the singlet methine in compound $\mathbf{1 0}$ has high field shifting $(\delta 4.96)$, which was not matching with our revealed spectral data, establishing the structure as 4,4'-diydroxypulvinone (7) [46].

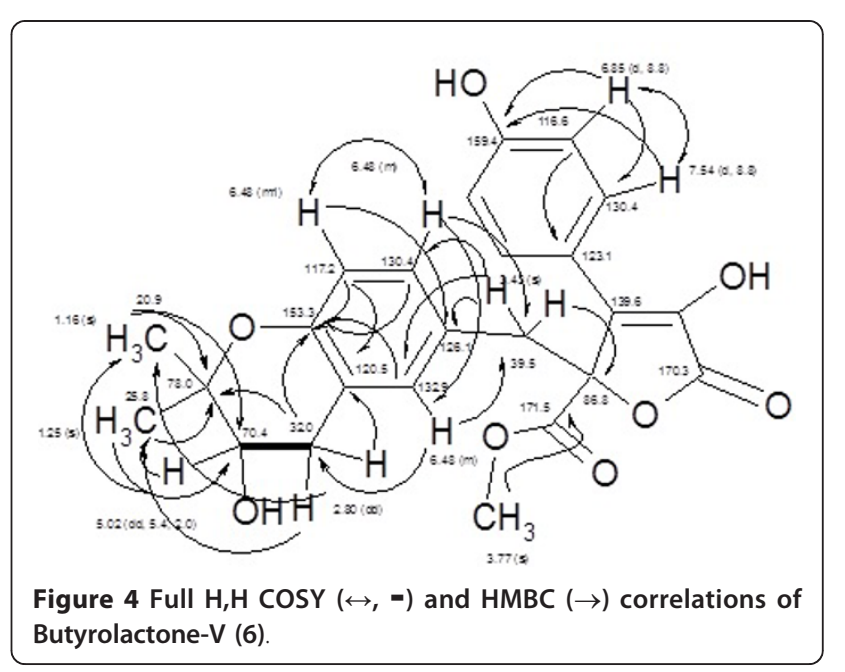




\subsection{Biological activities}

Activity patterns of the mycelial and supernatant extracts produced by fungal strain A. flavipes MM2 against set of microorganisms namely, Staphylococcus aureus, Pseudomonas aeruginosa, Candida albicans and Aspergillus niger were carried out using agar disc method $(25 \mu \mathrm{g} /$ disc, $\varnothing 5 \mathrm{~mm})$. In accordance, both extracts showed high antibacterial $(16-14 \mathrm{~mm})$ and moderate anti-yeast and antifungal $(10-14 \mathrm{~mm})$ activities (Table 1).

Alternatively, the isolated compounds 1-7 were tested against Bacillus subtilis, S. aureus, Streptomyces viridochromogenes (Tü 57), Escherichia coli, C. albicans, Mucor miehi, Chlorella vulgaris, Chlorella sorokiniana, Scenedesmus subspicatus, Rhizoctonia solani and Pythium ultimum $(40 \mu \mathrm{g} / \mathrm{disc}, \varnothing 9 \mathrm{~mm})$. According to this study, only four compounds $(\mathbf{1}, \mathbf{2}, \mathbf{4}, 7)$ were active. Ergosterol (1) was highly and moderately active against $S$. aureus and B. subtilis. Compounds 7, 4 and 3 showed high and moderate activity against $S$. aureus,. Finally, the whole studied compounds were tested against brine shrimp $(10 \mu \mathrm{g} / \mathrm{mL})$ for cytotoxic activities, exhibiting no cytotoxicity except ergosterol (1), which showed $100 \%$ cytotoxicity after $15 \mathrm{~h}$ (Table 2).

\section{Experimental}

NMR spectra were measured on Varian Unity 300 and Varian Inova 600 spectrometers. Electron spray ionization mass spectrometry (ESI HRMS): Finnigan LCQ ion trap mass spectrometer coupled with a Flux Instruments (Basel, Switzerland) quaternary pump Rheos 4000 and a HP 1100 HPLC (nucleosil column EC 125/2, 100-5, C 18) with autosampler (Jasco 851-AS, Jasco Inc., Easton, MD, USA) and a Diode Array Detector (Finnigan Surveyor LC System). High-resolution mass spectra (HRMS) were recorded by ESI MS on an Apex IV 7 Tesla Fourier-Transform Ion Cyclotron Resonance Mass Spectrometer (Bruker Daltonics, Billerica, MA, USA). EI MS at $70 \mathrm{eV}$ with Varian MAT 731, Varian 311A, AMD-402, high-resolution with perflurokerosine as standard. $R_{\mathrm{f}}$ values were measured on Polygram SIL F/ $\mathrm{UV}_{254}$ (Merck, pre-coated sheets). Size exclusion chromatography was done on Sephadex LH-20 (Pharmacia).

\subsection{Isolation and taxonomy of the producing strain}

The terrestrial $A$. flavipes MM2, which was identified according to the Raper and Fennel [14], has been isolated from rice hulls sample by placing the rice hulls over water agar medium (g/L): Agar-agar (20) and water (100\%) with incubation at $28^{\circ} \mathrm{C}$ for 7 days the developing colony was transferred to Czapeks agar with incubation at $28^{\circ} \mathrm{C}$ for 10 days. Bright whit-faint yellow colonies with a brown straining background of the fungal strain were grown. The colonies are growing rather slowly, showing whitish from conidial masses, with brownish conidiophores shining through, reverse yellowbrown to red brown Conidial heads spas, loosely columnar, conidiophores smooth-walled, pale yellow to light brown 2.4-3.2 $\mu \mathrm{m}$ diameter. According to its morphological and microscopic characteristics and comparison with literature, the fungal strain was assigned as $A$. flavipes MM2 [14]. The strain is deposited in Dr Mohammad Magdy El Metwally collection in Microbiology Department, Soil \& Water and Environment Research Institute, ARC, Giza, Egypt.

\subsection{Fermentation, extraction and isolation}

Small pieces $\left(1 \mathrm{~cm}^{2}\right)$ of well grown sub-cultures of $A$. flavipes MM2 were inoculated into thirty 1-L Erlenmeyer flasks, each containing $300 \mathrm{~mL}$ of sterilized Czapeck-Dox medium (g/L): Sucrose (30), $\mathrm{NaNO}_{3}$ (3), $\mathrm{K}_{2} \mathrm{HPO}_{4}(1), \mathrm{KCl}(0.5), \mathrm{MgSO}_{4}(0.5), \mathrm{FeSO}_{4}(0.01)$ and distilled water $(1 \mathrm{~L})$ at $\mathrm{pH}=(7.3)$. The inoculated flasks were incubated for 10 days at $28^{\circ} \mathrm{C}$ and $100 \mathrm{rpm}$. After harvesting, the fungal mate and supernatant were separated by filtration. The fungal mat was then applied to maceration in methanol $(3 \times 0.5 \mathrm{~L})$. The methanol extract was concentrated in vacuum and the remaining aqueous solution was re-extracted by ethyl acetate followed by concentration to yield $845 \mathrm{mg}$ as brown crude extract. The supernatant was passed through XAD-16 column $(4 \times 120 \mathrm{~cm})$. After washing with water, the absorbed organic extract was eluted by methanol, followed by concentration under vacuum, and the aqueous residue was re-extracted by ethyl acetate, followed by concentration in vacuo to afford $818 \mathrm{mg}$ as brown crude extract.

The mycelial extract ( $845 \mathrm{mg}$ ) was subjected to fractionation using silica gel column chromatography (cyclohexane- $\mathrm{CH}_{2} \mathrm{Cl}_{2}-\mathrm{MeOH}$ ) to afford $200 \mathrm{mg}$ as major fraction, which was then washed by $\mathrm{MeOH}$ to deliver a colourless precipitate. The last precipitate was purified on Sephadex LH-20 $\left(\mathrm{CH}_{2} \mathrm{Cl}_{2} / 40 \% \mathrm{MeOH}\right)$ to afford Ergosterol (1, $12 \mathrm{mg})$ as colourless solid.

Table 1 Pre-antimicrobial assays of A. flavipes MM2 ( $\varphi \mathrm{mm})$

\begin{tabular}{|c|c|c|c|c|c|c|c|c|}
\hline \multirow[t]{3}{*}{ Medium no. } & \multicolumn{8}{|c|}{ Inhibition zone (mm) } \\
\hline & \multicolumn{4}{|c|}{ Culture filtrate extract } & \multicolumn{4}{|l|}{ Cells extract } \\
\hline & $\begin{array}{c}\text { St. } \\
\text { aureus }\end{array}$ & Ps. aeruginosa, & C. albicans & A. niger & St. Aureus & Ps. aeruginosa, & C. albicans & A. niger \\
\hline Czapeck-Dox & 15 & 16 & 14 & 12 & 14 & 14 & 10 & 10 \\
\hline
\end{tabular}


Table 2 Antimicrobial assays of A. flavipes MM2 compounds ( $\varphi \mathrm{mm}$ )

\begin{tabular}{lcccccccccccc}
\hline Compound (No.) & \multicolumn{10}{c}{ Inhibition zone (mm) of tested microorganisms } \\
\cline { 2 - 11 } & $\begin{array}{c}\text { B. } \\
\text { sub. }\end{array}$ & $\begin{array}{c}\text { St. } \\
\text { aur }\end{array}$ & $\begin{array}{c}\text { St. } \\
\text { Virid }\end{array}$ & $\begin{array}{c}\text { E. } \\
\text { coli }\end{array}$ & $\begin{array}{c}\text { C. } \\
\text { alb }\end{array}$ & M. miehi & $\begin{array}{c}\text { Ch. } \\
\text { vulg }\end{array}$ & Ch. Sorok & $\begin{array}{c}\text { Sce. } \\
\text { sub }\end{array}$ & $\begin{array}{c}\boldsymbol{R} \text {. } \\
\text { solani }\end{array}$ & $\begin{array}{c}\boldsymbol{P} \text { ultim } \\
\text { Brine shrimp }\end{array}$ \\
\hline Ergosterol (1) & 11 & 19 & ND & ND & ND & ND & ND & ND & ND & ND & ND & $100 \%$ \\
Butyrolactone I (2) & ND & ND & ND & ND & ND & ND & ND & ND & ND & ND & ND & ND \\
Aspulvinone H (3) & ND & 11 & ND & ND & ND & ND & ND & ND & ND & ND & ND & ND \\
6-methylsalicylic acid (4) & ND & 15 & ND & ND & ND & ND & ND & ND & ND & ND & ND & ND \\
Terrien (5) & ND & ND & ND & ND & ND & ND & ND & ND & ND & ND & ND & ND \\
Butyrolactone-V (6) & ND & ND & ND & ND & ND & ND & ND & ND & ND & ND & ND & ND \\
4,4'-diydroxypulvinone (7) & ND & 18 & ND & ND & ND & ND & ND & ND & ND & ND & ND & ND \\
\hline
\end{tabular}

The filtrate crude extract $(818 \mathrm{mg})$ was fractionated on silica gel column and eluted by cyclohexane- $\mathrm{CH}_{2} \mathrm{Cl}_{2}$ $\mathrm{MeOH}$ gradient to give five fractions (I-V). Fraction FIII was further fractionated using Sephadex LH-20 column $(\mathrm{MeOH})$ to afford two sub-fractions, FIIIa (44 mg) and FIIIb $(73 \mathrm{mg})$. Purification of FIIIa by Sephadex LH-20 $(\mathrm{MeOH})$ afforded a colourless solid of butyrolactone-I (2, $4 \mathrm{mg})$. PTLC purification $\left(\mathrm{CH}_{2} \mathrm{Cl}_{2} / 5 \% \mathrm{MeOH}\right)$ of FIIIb followed by Sephadex LH-20 (MeOH) afforded Aspulvinone $\mathrm{H}(3)$ as colourless solid (3 mg). A further fractionation of FIV on Sephadex LH-20 (MeOH) led to sub-fractions FIVa $(35 \mathrm{mg})$ and FIVb $(120 \mathrm{mg})$. Purification of sub-fraction FIVa using Sephadex LH-20 $(\mathrm{MeOH})$, PTLC $\left(\mathrm{CH}_{2} \mathrm{Cl}_{2} / 5 \% \mathrm{MeOH}\right)$, and then Sephadex LH-20 (MeOH) resulted in 6-methylsalicylic acid (4, $10 \mathrm{mg}$ ) as colourless solid. Sub-fraction FIVb was purified on Sephadex LH-20 (MeOH) to afford Terrien (5, $10 \mathrm{mg})$ and butyrolactone- $\mathrm{V}(\mathbf{6}, 2 \mathrm{mg})$ as two colourless solids. Finally, fraction FV was purified using two subsequent Sephadex LH-20 columns $(\mathrm{MeOH})$ to give a yellow solid of 4,4'-diydroxypulvinone (7, $4 \mathrm{mg})$.

\subsubsection{Ergosterol; ergosta-5,7,22-triene-3 $\beta$-ol (1)}

$\mathrm{C}_{28} \mathrm{H}_{44} \mathrm{O}$ (396), colourless solid, UV-absorbing, turned violet with anisaldehyde/sulphuric acid, $R_{\mathrm{f}}=0.46$ $\left(\mathrm{CH}_{2} \mathrm{Cl}_{2} / 5 \% \mathrm{MeOH}\right) ;{ }^{1} \mathbf{H}$ NMR $\left(\mathrm{CDCl}_{3}, 300 \mathrm{MHz}\right): \delta=$ $5.57(\mathrm{dm}, 1 \mathrm{H}, \mathrm{H}-6), 5.38(\mathrm{dm}, 1 \mathrm{H}, \mathrm{H}-7), 5.17(\mathrm{~m}, 2 \mathrm{H}$, H-22,23), 3.62 (m, 1H, H-3), 2.46 (dm, 1H, H-5), 2.35 (m, 2H, H-20, 24), 2.09-1.93 (m, 3H), 1.92-1.89 (m, 4H), $1.88-1.55(\mathrm{~m}, 4 \mathrm{H}), 1.50-1.40(\mathrm{~m}, 3 \mathrm{H}), 1.38-1.16(\mathrm{~m}, 3 \mathrm{H})$, 1.02 (d, $J=7.2,3 \mathrm{H}, \mathrm{CH}_{3}-21$ ), 0.93 (s, 3H, $\left.\mathrm{CH}_{3}-19\right), 0.91$ (d, $\left.J=7.2,3 \mathrm{H}, \mathrm{CH}_{3}-28\right), 0.82\left(\mathrm{~d}, J=6.8,3 \mathrm{H}, \mathrm{CH}_{3}-27\right)$, $0.80\left(\mathrm{~d}, J=6.8,3 \mathrm{H}, \mathrm{CH}_{3}-26\right), 0.61\left(\mathrm{~s}, 3 \mathrm{H}, \mathrm{CH}_{3}-18\right) ;{ }^{13} \mathrm{C}$ NMR $\left(\mathrm{CDCl}_{3}, 75 \mathrm{MHz}\right): \delta=141.3\left(\mathrm{C}_{\mathrm{q}}-8\right), 139.8\left(\mathrm{C}_{\mathrm{q}}-5\right)$, 135.5 (CH-22), 131.9 (CH-23), 119.6 (CH-7), $116.3(\mathrm{CH}-$ 6), $70.4(\mathrm{CH}-3), 55.7(\mathrm{CH}-17), 54.5(\mathrm{CH}-14), 46.2(\mathrm{CH}-$ 9), $42.8\left(\mathrm{C}_{\mathrm{q}}-13\right), 42.8(\mathrm{CH}-24), 40.7\left(\mathrm{CH}_{2}-4\right), 40.4(\mathrm{CH}-$ 20), $39.1\left(\mathrm{CH}_{2}-12\right), 38.4\left(\mathrm{CH}_{2}-1\right), 37.0\left(\mathrm{C}_{\mathrm{q}}-10\right), 33.1$ (CH-25), $32.0\left(\mathrm{CH}_{2}-2\right), 28.3\left(\mathrm{CH}_{2}-16\right), 23.0\left(\mathrm{CH}_{2}-11\right)$, $21.1\left(\mathrm{CH}_{2}-15\right), 21.1\left(\mathrm{CH}_{3}-21\right), 19.9\left(\mathrm{CH}_{3}-27\right), 19.6\left(\mathrm{CH}_{3}-\right.$ 26), $17.6\left(\mathrm{CH}_{3}-28\right), 16.2\left(\mathrm{CH}_{3}-19\right), 12.0\left(\mathrm{CH}_{3}-18\right)$; EIMS (70 EV): $m / z(\%)=396\left([\mathrm{M}]^{+}, 87\right), 378\left(\left[\mathrm{M}-\mathrm{H}_{2} \mathrm{O}\right]^{+}\right.$,
12), $363\left(\left[\mathrm{M}-\left(\mathrm{H}_{2} \mathrm{O}+\mathrm{CH}_{3}\right)\right]^{+}, 100\right), 271$ (25), 253 (52), 211 (33).

\subsubsection{Butyrolactone-I (2)}

$\mathrm{C}_{24} \mathrm{H}_{24} \mathrm{O}_{7}$ (424), colourless solid, UV-absorbing, turned violet with anisaldehyde/sulphuric acid, $R_{\mathrm{f}}=0.39$ $\left(\mathrm{CH}_{2} \mathrm{Cl}_{2} / 5 \% \mathrm{MeOH}\right) ;{ }^{1} \mathbf{H}$ NMR $\left(\mathrm{CD}_{3} \mathrm{OD}, 300 \mathrm{MHz}\right): \delta=$ $7.57\left(\mathrm{~d}, J=8.8 \mathrm{~Hz}, 2 \mathrm{H}, \mathrm{H}-2^{\prime}, 6^{\prime}\right), 6,86(\mathrm{~d}, J=8.8 \mathrm{~Hz}, 2 \mathrm{H}$, H-3',5'), 6.50 (dd, $J=7.1,2.0 \mathrm{~Hz}, 1 \mathrm{H}, \mathrm{H}-6$ "), 6.48 (d, $J=$ $8.1 \mathrm{~Hz}, 1 \mathrm{H}, \mathrm{H}-5$ "), 6.40 (d, $J=1.7,1 \mathrm{H}, \mathrm{H}-2$ )), 5.05 (t, $J$ $=3.7 \mathrm{~Hz}, 1 \mathrm{H}, \mathrm{H}-8$ "), $3.76\left(\mathrm{~s}, 3 \mathrm{H}, 7-\mathrm{OCH}_{3}\right), 3.42(\mathrm{~d}, J=$ 3.9, 2H, $\mathrm{CH}_{2}-5$ ), 3.06 (d, $J=7.3 \mathrm{~Hz}, 2 \mathrm{H}, \mathrm{CH}_{2}-7$ "), 1.65 (s, $3 \mathrm{H}, \mathrm{CH}_{3}-10$ "), 1.56 (s, $3 \mathrm{H}, \mathrm{CH}_{3}-11$ "); ${ }^{13} \mathrm{C}$ NMR $\left(\mathrm{CD}_{3} \mathrm{OD}, 75 \mathrm{MHz}\right): \delta=171.6\left(\mathrm{C}_{\mathrm{q}}-6\right), 170.3\left(\mathrm{C}_{\mathrm{q}}-1\right), 159.3$ $\left(\mathrm{C}_{\mathrm{q}}-4^{\prime}\right), 155.0\left(\mathrm{C}_{\mathrm{q}}-4^{\prime \prime}\right), 139.6\left(\mathrm{C}_{\mathrm{q}}-3\right), 133.0\left(\mathrm{C}_{\mathrm{q}}-9^{\prime \prime}\right), 132.4$ (CH-2"), 130.4 (CH-2',6'), 129.8 (CH-6"), $129.2\left(\mathrm{C}_{\mathrm{q}}-3^{\prime \prime}\right)$, $125.0\left(\mathrm{C}_{\mathrm{q}}-1^{\prime \prime}\right), 123.1\left(\mathrm{C}_{\mathrm{q}}-1^{\prime}\right), 123.6(\mathrm{CH}-8$ "), $116.6(\mathrm{CH}-$ 3',5'), $115.0\left(\mathrm{CH}-5^{\prime \prime}\right), 86.8\left(\mathrm{C}_{\mathrm{q}}-4\right), 53.8\left(\mathrm{OCH}_{3}-7\right), 39.6$ ( $\left.\mathrm{CH}_{2}-5\right), 28.7\left(\mathrm{CH}_{2}-7\right.$ "), $25.9\left(\mathrm{CH}_{3}-10 "\right), 17.8\left(\mathrm{CH}_{3}-11\right.$ "); $-(+)$ ESI MS: $m / z(\%)=447\left([\mathrm{M}+\mathrm{Na}]^{+}, 81\right), 871([2 \mathrm{M}$ $\left.+\mathrm{Na}]^{+}, 100\right)$; -(-)ESI MS: $m / z(\%)=423\left([\mathrm{M}-\mathrm{H}]^{-}, 13\right)$, $847\left([2 \mathrm{M}-\mathrm{H}]^{-}, 4\right) ;(+)$-HRESI: $m / z=447.1414[\mathrm{M}+\mathrm{Na}]^{+}$ (calc. 447.1414 for $\mathrm{C}_{24} \mathrm{H}_{24} \mathrm{NaO}_{7}$ ); (-)-HRESI: $\mathrm{m} / z$ $423.1435[\mathrm{M}-\mathrm{H}]^{-}$(calc. 423.1449 for $\mathrm{C}_{24} \mathrm{H}_{23} \mathrm{O}_{7}$ ).

\subsubsection{Aspulvinone $\mathrm{H}$ (3)}

$\mathrm{C}_{27} \mathrm{H}_{28} \mathrm{O}_{5}$ (432), colourless solid, UV-blue fluorescent, turned yellow with anisaldehyde/sulphuric acid, $R_{\mathrm{f}}=$ $0.62\left(\mathrm{CH}_{2} \mathrm{Cl}_{2} / 10 \% \mathrm{MeOH}\right),{ }^{1} \mathbf{H}$ NMR $\left(\mathrm{CD}_{3} \mathrm{OD}, 300\right.$ $\mathrm{MHz}): \delta=7.81\left(\mathrm{~d}, J=1.8 \mathrm{~Hz}, 1 \mathrm{H}, \mathrm{H}-2^{\prime}\right), 7.68(\mathrm{~m}, 1 \mathrm{H}$, H-6'), 7.59 (m, 1H, H-6"), 7.44 (d, $\left.J=1.6 \mathrm{~Hz}, \mathrm{H}-2^{\prime \prime}\right)$, $6.73\left(\mathrm{~m}, 2 \mathrm{H}, \mathrm{H}-5^{\prime}, 5^{\prime \prime}\right), 6.22(\mathrm{~s}, \mathrm{H}-5), 5.36(\mathrm{~m}, 2 \mathrm{H}, \mathrm{H}-$ 8', 8"), 3.40-3.00 (m, 4H, $\left.\mathrm{H}_{2 \mathrm{a}, \mathrm{b}^{-}} 7^{\prime}, \mathrm{H}_{2 \mathrm{a}, \mathrm{b}}-7^{\prime \prime}\right), 1.75$ (m, $\left.12 \mathrm{H}, \mathrm{H}_{3}-10^{\prime}, 11^{\prime}, 10^{\prime \prime}, 11^{\prime \prime}\right) ;-(+)$ ESI MS: $m / z(\%)=455([\mathrm{M}$ $\left.+\mathrm{Na}]^{+}, 56\right), 477\left([\mathrm{M}+2 \mathrm{Na}-\mathrm{H}]^{+}, 100\right), 887\left([2 \mathrm{M}+\mathrm{Na}]^{+}, 5\right)$; -(-)ESI MS: $m / z(\%)=431\left([\mathrm{M}-\mathrm{H}]^{-}, 100\right), 863\left([2 \mathrm{M}-\mathrm{H}]^{-}\right.$, 4), (+)-HRESI: $m / z 455.1808[\mathrm{M}+\mathrm{Na}]^{+}$(calc. 455.1829 for $\mathrm{C}_{27} \mathrm{H}_{28} \mathrm{NaO}_{5}$ ); (-)-HRESI: $m / z 431.1860[\mathrm{M}-\mathrm{H}]^{-}$ (calc. 431.1864 for $\mathrm{C}_{27} \mathrm{H}_{27} \mathrm{O}_{5}$ ).

\subsubsection{6-Methylsalicylic acid (4)}

$\mathrm{C}_{8} \mathrm{H}_{8} \mathrm{O}_{3}$ (152), colourless solid, UV-absorbing, $R_{\mathrm{f}}=0.24$ $\left(\mathrm{CH}_{2} \mathrm{Cl}_{2} / 5 \% \mathrm{MeOH}\right) ;{ }^{1} \mathbf{H}$ NMR $\left(\mathrm{CD}_{3} \mathrm{OD}, 300 \mathrm{MHz}\right): \delta=$ $7.07(\mathrm{t}, J=7.7 \mathrm{~Hz}, 1 \mathrm{H}, 4-\mathrm{H}), 6.63(\mathrm{~d}, J=8.2 \mathrm{~Hz}, 1 \mathrm{H}, 3-$ 
$\mathrm{H}), 6.59(\mathrm{~d}, J=7.5 \mathrm{~Hz}, 1 \mathrm{H}, 5-\mathrm{H}), 2.56\left(\mathrm{~s}, 3 \mathrm{H}, \mathrm{CH}_{3}-7\right)$; ${ }^{13} \mathrm{C}$ NMR (CD $\left.3 \mathrm{OD}, 75 \mathrm{MHz}\right): \delta=176\left(\mathrm{C}_{\mathrm{q}}-8\right), 162.7$ $\left(\mathrm{C}_{\mathrm{q}}-2\right), 142.7\left(\mathrm{C}_{\mathrm{q}}-6\right), 132.1(\mathrm{CH}-4), 122.9(\mathrm{CH}-3), 119.5$ $\left(\mathrm{C}_{\mathrm{q}}-1\right)$, 115.1 (CH-5), $23.3\left(\mathrm{CH}_{3}-7\right)$; $-(+)$ ESI MS: $m / z(\%)$ $=175\left([\mathrm{M}+\mathrm{Na}]^{+}, 25\right), 371\left([2 \mathrm{M}+3 \mathrm{Na}-2 \mathrm{H}]^{+}, 55\right) ;-(-) \mathbf{E S I}$ MS: $m / z(\%)=151\left([\mathrm{M}-\mathrm{H}]^{-}, 100\right), 303\left([2 \mathrm{M}-\mathrm{H}]^{-}, 4\right)$.

\subsubsection{Terrine (5)}

$\mathrm{C}_{8} \mathrm{H}_{10} \mathrm{O}_{3}$ (154), colourless solid, UV-absorbing, turned dark green on spraying with anisaldehyde/sulphuric acid, $R_{\mathrm{f}}=0.51\left(\mathrm{CH}_{2} \mathrm{Cl}_{2} / 10 \% \mathrm{MeOH}\right) ;{ }^{1} \mathbf{H}$ NMR $\left(\mathrm{CD}_{3} \mathrm{OD}\right.$, $300 \mathrm{MHz}): \delta=6.82(\mathrm{ddd}, J=13.7,8.9,6.8 \mathrm{~Hz}, 1 \mathrm{H}, \mathrm{H}-$ 2), 6.42 (dd, $J=15.8,1.1 \mathrm{~Hz}, 1 \mathrm{H}, \mathrm{H}-3), 5.99$ (s, $1 \mathrm{H}, \mathrm{H}-$ 5), 4.67 (d, $J=2.4 \mathrm{~Hz}, 1 \mathrm{H}, \mathrm{H}-8), 4.07$ (d, $J=2.7 \mathrm{~Hz}, 1 \mathrm{H}$, $\mathrm{H}-7), 1.97\left(\mathrm{dd}, J=6.8,1.4 \mathrm{~Hz}, 3 \mathrm{H}, 1-\mathrm{CH}_{3}\right) ;{ }^{13} \mathrm{C}$ NMR $\left(\mathrm{CD}_{3} \mathrm{OD}, 75 \mathrm{MHz}\right): \delta=205.6\left(\mathrm{C}_{\mathrm{q}}-6\right), 170.8\left(\mathrm{C}_{\mathrm{q}}-4\right), 141.8$ (CH-2), 126.4 (CH-3), $125.9(\mathrm{CH}-5), 82.4(\mathrm{CH}-7), 78.1$ $(\mathrm{CH}-8), 19.5\left(\mathrm{CH}_{3}-1\right)$; $-(+)$ ESI MS: $m / z(\%)=177([\mathrm{M}$ $\left.+\mathrm{Na}]^{+}, 62\right), 331\left([2 \mathrm{M}+\mathrm{Na}]^{+}, 100\right)$; $-(-)$ ESI MS: $m / z(\%)=$ $153\left([\mathrm{M}-\mathrm{H}]^{-}, 34\right), 307$ ([2M-H] $\left.]^{-}, 4\right) ;(+)$-HRESI MS: $m / z$ $177.0528[\mathrm{M}+\mathrm{Na}]^{+}$(calc. 177.0522 for $\mathrm{C}_{8} \mathrm{H}_{10} \mathrm{NaO}_{3}$ ); (-)-HRESI MS: $m / z 153.0553[\mathrm{M}-\mathrm{H}]^{-}$(calc. 153.0557 for $\left.\mathrm{C}_{8} \mathrm{H}_{9} \mathrm{O}_{3}\right)$.

\subsubsection{Butyrolactone-V (6)}

$\mathrm{C}_{24} \mathrm{H}_{24} \mathrm{O}_{8}$ (440), colourless solid, UV-absorbing, turned pink with anisaldehyde/sulphuric acid, $R_{\mathrm{f}}=0.12$ $\left(\mathrm{CH}_{2} \mathrm{Cl}_{2} / 5 \% \mathrm{MeOH}\right),{ }^{\mathbf{1}} \mathbf{H}$ NMR $\left(\mathrm{CD}_{3} \mathrm{OD}, 300 \mathrm{MHz}\right): \delta=$ $7.54\left(\mathrm{~d}, J=8.8 \mathrm{~Hz}, 2 \mathrm{H}, \mathrm{H}-2^{\prime}, 6^{\prime}\right), 6.85(\mathrm{~d}, J=8.8 \mathrm{~Hz}, 2 \mathrm{H}$, H-3',5'), 6.48 (m, 3H, H-2",5”,6”), 5.02 (dd, $J=5.2,2.0$ $\mathrm{Hz}, 1 \mathrm{H}, \mathrm{H}-8$ "), 3.77 (s, 3H, $\left.\mathrm{OCH}_{3}-7\right), 3.40$ (s, 2H, $\mathrm{CH}_{2}-$ 5), 2.80 (dd, $J=5.2,16.9 \mathrm{~Hz}, 2 \mathrm{H}, \mathrm{CH}_{2}-7$ )), 1.25 (s, $3 \mathrm{H}$, $\mathrm{CH}_{3}-10$ "), 1.16 (s, 3H, $\mathrm{CH}_{3}-11$ "); ${ }^{13} \mathrm{C}$ NMR ( $\mathrm{CD}_{3} \mathrm{OD}, 75$ $\mathrm{MHz}): \delta=171.5\left(\mathrm{C}_{\mathrm{q}}-6\right), 170.3\left(\mathrm{C}_{\mathrm{q}}-1\right), 159.4\left(\mathrm{C}_{\mathrm{q}}-4^{\prime}\right)$, 153.3 ( $\mathrm{C}_{\mathrm{q}}-4$ "), $139.6\left(\mathrm{C}_{\mathrm{q}}-3\right), 132.9$ (CH-2"), $130.4(\mathrm{CH}-$ 2',6'), 130.4 (CH-6"), $120.5\left(\mathrm{C}_{\mathrm{q}}-3^{\prime \prime}\right), 126.0\left(\mathrm{C}_{\mathrm{q}}-1^{\prime \prime}\right), 123.1$ $\left(\mathrm{C}_{\mathrm{q}}-1^{\prime}\right), 116.6\left(\mathrm{CH}-3^{\prime}, 5^{\prime}\right), 117.2\left(\mathrm{CH}-5^{\prime \prime}\right), 86.8\left(\mathrm{C}_{\mathrm{q}}-4\right), 78.0$ ( $\mathrm{C}_{\mathrm{q}}-9$ "), $70.4\left(\mathrm{CH}-8\right.$ "), $53.9\left(\mathrm{OCH}_{3}-7\right), 39.5\left(\mathrm{CH}_{2}-5\right), 32.0$ ( $\mathrm{CH}_{2}-7$ "), 25.8 ( $\mathrm{CH}_{3}-10$ "), $20.9\left(\mathrm{CH}_{3}-11\right.$ "); -(+)ESI MS: $m / z(\%)=441\left([\mathrm{M}+\mathrm{H}]^{+}, 30\right), 463\left([\mathrm{M}+\mathrm{Na}]^{+}, 57.5\right), 881$ $\left([2 \mathrm{M}+\mathrm{H}]^{+}, 25\right), 903\left([2 \mathrm{M}+\mathrm{Na}]^{+}, 50\right)$; -(-)ESI MS: $m / z$ (\%) 439 ([M-H] $\left.]^{-}, 3\right)$; (+)-HRESI: $m / z 463.1370[\mathrm{M}+\mathrm{Na}]^{+}$ (calc. 463.1363 for $\mathrm{C}_{24} \mathrm{H}_{24} \mathrm{NaO}_{8}$ ); (-)-HRESI: $\mathrm{m} / z$ 439.1399 [M-H] $]^{-}$(calc. 439.1389 for $\mathrm{C}_{24} \mathrm{H}_{23} \mathrm{O}_{8}$ ).

\subsubsection{4,4'-Diydroxypulvinone (7)}

$\mathrm{C}_{17} \mathrm{H}_{12} \mathrm{O}_{5}$ (296), colourless solid, UV yellow fluorescence, $R_{\mathrm{f}}=0.15\left(\mathrm{CH}_{2} \mathrm{Cl}_{2} / 5 \% \mathrm{MeOH}\right) ;{ }^{1} \mathbf{H} \mathbf{N M R}$ (DMSO-d $6,300 \mathrm{MHz}$ ): $\delta=9.42$ (brs, $\left.1 \mathrm{H}, 4{ }^{\prime}-\mathrm{OH}\right), 8.70$ (brs, 1H, 4"-OH), 8.06 (d, $J=8.6 \mathrm{~Hz}, 2 \mathrm{H}, \mathrm{H}-2^{\prime}, 6$ '), 7.51 (d, $J=8.7 \mathrm{~Hz}, 2 \mathrm{H}, \mathrm{H}-2$ ",6"), 6.75 (d, $J=8.7 \mathrm{~Hz}, 2 \mathrm{H}, \mathrm{H}-$ 3', 5'), 6.16 (d, J = 8.7 Hz, 2H, H-3”, 5”), 5.88 (s, 1H, H5); ${ }^{13} \mathrm{C}$ NMR (DMSO- $\left.\mathrm{d}_{6}, 300 \mathrm{MHz}\right): \delta=156.1\left(\mathrm{C}_{\mathrm{q}^{-}}\right.$ 4',4”), $152.4\left(\mathrm{C}_{\mathrm{q}}-4\right), 130.4\left(\mathrm{CH}-2^{\prime}, 2^{\prime \prime}, 6^{\prime}, 6^{\prime \prime}\right), 126.1\left(\mathrm{C}_{\mathrm{q}}-1^{\prime}\right.$, 1"), 124.6 (CH-5), 115.3 (CH-3',5'), 114.1 (CH-3",5”); $-(+)-$ ESI MS: $m / z(\%)=319\left([\mathrm{M}+\mathrm{Na}]^{+}, 20\right), 314([\mathrm{M}$ $\left.+2 \mathrm{Na}-\mathrm{H}]^{+}, 30\right)$; -(-)ESI MS: $m / z(\%)=295\left([\mathrm{M}-\mathrm{H}]^{-}\right.$,
100); (+)-HRESI MS: $m / z 319.0587[\mathrm{M}+\mathrm{Na}]^{+}$(calc. 319.0577 for $\mathrm{C}_{17} \mathrm{H}_{12} \mathrm{NaO}_{5}$ ). (-)-HRESI: $m / z 295.0616$ [M-H] $]^{-}$(calc. 295.0612 for $\mathrm{C}_{17} \mathrm{H}_{11} \mathrm{O}_{5}$ ).

\subsection{Biological activities \\ 3.3.1. Antimicrobial activity}

Antimicrobial assays were conducted utilizing the discagar method [47]. This has been carried out gainst diverse sets of microorganisms. A. flavipes MM2 extract was dissolved in $\mathrm{CH}_{2} \mathrm{Cl}_{2} / 10 \% \mathrm{MeOH}$ at a concentration of $1 \mathrm{mg} / \mathrm{mL}$. Aliquots of $40 \mu \mathrm{L}$ were soaked on filter paper discs (9 mm $\varnothing$, no. 2668, Schleicher \& Schüll, Germany) and dried for $1 \mathrm{~h}$ at room temperature under sterilized conditions. The paper discs were placed on inoculated agar plats and incubated for $24 \mathrm{~h}$ at $38^{\circ} \mathrm{C}$ for bacterial and $48 \mathrm{~h}\left(30^{\circ} \mathrm{C}\right)$ for the fungal isolates, while the algal test strains were incubated at approximately $22^{\circ} \mathrm{C}$ in day light for 8-10 days. The pure compounds were examined against the test microorganisms: $B$. subtilis, S. aureus, S. viridochromogenes (Tü 57), E. coli, C. albicans, M. miehi, C. vulgaris, C. sorokiniana, S. subspicatus, $R$. solani and P. ultimum.

\subsubsection{Brine shrimp microwell cytotoxicity assay}

The cytotoxic assay was performed according to Sajid et al.'s screening [48].

\section{Acknowledgements}

The authors are deeply thankful to Prof. H. Laatsch for his Lab facilities and unlimited support. We thank Dr. H. Frauendorf and Mr. R. Machinek for the spectral measurements. We appreciate to Miss F. Lissy for testing biological activity and Mr. A. Kohl for his technical assistance. This research work is financed by German Egyptian Scientific Projects (GESP) No. 7.

\section{Author details}

${ }^{1}$ Division of Pharmaceutical Industries, Chemistry of Natural Compounds Department, National Research Centre, El-Behoos st. 33, Dokki, Cairo 12622, Egypt ${ }^{2}$ Microbial Activity Unit, Microbiology Department, Soil \& Water and Environment Research Institute, ARC, Giza, Egypt ${ }^{3}$ Institute of Organic and Biomolecular Chemistry, University of Göttingen, Tammannstrasse 2, D-37077 Göttingen, Germany ${ }^{4}$ Pharmacognosy Department, Faculty of Pharmacy, Cairo University, Cairo, Egypt

\section{Competing interests}

The authors declare that they have no competing interests.

Received: 18 November 2011 Accepted: 1 March 2012 Published: 1 March 2012

\section{References}

1. Faulkner DJ (2006) Marine bacterial metabolites. In: Proksch P, Muller WEG (eds) Frontiers in marine biotechnology. Horizon Bioscience pp 225-288

2. Laatsch $H$ (2010) A data base for rapid structural determination of microbial natural products and annual updates. Chemical Concepts, Weinheim, Germany

3. Larsen TO, Smedsgaard J, Nielsen KF, Hansen ME, Frisvad JC (2005) Phenotypic taxonomy and metabolite profiling in microbial drug discovery. Nat Prod Rep 22:672-695. doi:10.1039/b404943h.

4. Hassan AEHA (2007) Novel natural products from endophytic fungi of Egyptian medicinal plants-chemical and biological characterization. Dissertation, Düsseldorf, Germany

5. Butler MS (2004) The role of natural product chemistry in drug discovery. J Nat Prod 67:2141-2153. doi:10.1021/np040106y. 
6. Rasmussen TB, Skinderso ME, Bjarnsholt T, Phipps RK, Christensen KB, Andersen JB, Koch B, Larsen TO, Hentzer M, Hoiby N, Givskov M (2005) Identity and effects of quorum-sensing inhibitors produced by Penicillium species. Microbiology 151:1325-1340. doi:10.1099/mic.0.27715-0.

7. Li JY, Strobel GA (2001) Jesterone and hydroxyjesterone antioomycete cyclohexenenone epoxides from the endophytic fungus: Pestalotiopsis jesteri. Phytochemistry 57:261-265. doi:10.1016/S0031-9422(01)00021-8.

8. Brady SF, Clardy J (2000) CR377, a new pentaketide antifungal agent isolated from an endophytic fungus. J Nat Prod 63:1447-1448. doi:10.1021/ np990568p.

9. Singh SB, Zink DL, Guan Z, Collado J, Pelaez F, Felock PJ, Hazuda DJ (2003) Isolation, structure and HIV-1 integrase inhibitory activity of xanthoviridicatin $\mathrm{E}$ and $\mathrm{F}$ two novel fungal metabolites produced by Penicillium chrysogenum. Helv Chim Acta 86:3380-3385. doi:10.1002/hlca.200390281.

10. Zhang HW, Song YC, Tan RX (2006) Biology and chemistry of endophytes. Nat Prod Rep 23:753-771. doi:10.1039/b609472b.

11. Song YC, Li H, Ye YH, Shan CY, Yang YM, Tan RX (2004) Endophytic naphthopyrone metabolites are co-inhibitors of xanthine oxidase, SW1116 cell and some microbial growths. FEMS Microbiol Lett 241:67-72. doi:10.1016/j.femsle.2004.10.005

12. Lubertozzi D, Keasling JD (2009) Developing Aspergillus as a host for heterologous expression. Biotechnol Adv 27:53-75. doi:10.1016/j. biotechadv.2008.09.001.

13. Zhang Z (2009) A new species of Aspergillus. Int J Biol 1:78-80

14. Raper KB, Fennel DI (1965) The genus Aspergillus. Williams and Wilkins Baltimore. USA

15. Liebermann C (1885) Ueber das oxychinoterpen, Berichte. 18:1803-1807

16. Mourao F, Umeo SH, Takemura OS, Linde GA, Colauto NB (2011) Antioxidant activity of Agaricus brasiliensis basidiocarps on different maturation phases. Braz J Microbiol 42:197-202. doi:10.1590/S151783822011000100024

17. Dissanayake DP, Abeytunga DTU, Vasudewa NS, Ratnasooriya WD (2009) Inhibition of lipid peroxidation by extracts of Pleurotus ostreatus. Pharmacogn Mag 5:266-271

18. Chen A, Chen H, Shao Y, Fan M (2009) Active components and free radical scavenging activity of fermented mycelia and broth of Paecilomyces tenuipes. Shipin Kexue 30:25-28

19. Han X, Lin Z, Tao H, Liu P, Wang Y, Zhu W (2009) Cytotoxic metabolites from symbiotic fungus Penicillium sp. HK13-8 with Rhizophora stylosa. Zhongguo Haiyang Yaowu 28:11-16

20. Yan D, Bao HY, Bau T, Li Y, Kim YH (2009) Antitumor components from Naematoloma fasciculare. J Microbiol Biotechnol 19:1135-1138

21. Nitta K, Fujita N, Yoshimura T, Arai K, Yamamoto U (1983) Metabolic products of Aspergillus terreus. IX: biosynthesis of butyrolactone derivatives isolated from strain IFO 8835 and 4100. Chem Pharm Bull 31:1528-1533. doi:10.1248/cpb.31.1528.

22. Zain ME, Awaad AS, Al-Jaber NA, Maitland DJ (2008) New phenolic compounds with antifungal activity from Aspergillus terreus isolated from desert soil. J Saudi Chem Soc 12:107-113

23. Nuclear P, Sommit D, Boonyuen N, Pudhom K (2010) Butenolide and furandione from an endophytic Aspergillus terreus. Chem Pharm Bull 58:1221-1223. doi:10.1248/cpb.58.1221.

24. Rao KV, Sadhukhan AK, Veerender M, Ravikumar V, Mohan EVS (2000) Butyrolactones from Aspergillus terreus. Chem Pharm Bull 48:559-562. doi:10.1248/cpb.48.559.

25. Parvatkar RR, Souza CD, Tripathi A, Naik CG (2009) Aspernolides \&Alpha; and \&Beta; butenolides from a marine-derived fungus. Aspergillus terreus. Phytochemistry 70:128-132

26. Kitagawa M, Okabe T, Ogino H, Matsumoto H, Suzuki-Takahashi I (1993) Butyrolactone I, a selective inhibitor of cdk2 and cdc2 kinase. Oncogene 8:2425-2432

27. Someya A, Tanaka N, Okuyama A (1994) Inhibition of cell cycle oscillation of DNA replication by a selective inhibitor of the cdc2 kinase family, butyrolactone I, in Xenopus egg extracts. Biochem Biophys Res Commun 198:536-545. doi:10.1006/bbrc.1994.1079.

28. Kitagawa M, Higashi H, Takahashi IS, Okabe T, Ogino H (1994) A cyclindependent kinase inhibitor, butyrolactone I, inhibits phosphorylation of RB protein and cell cycle progression. Oncogene 9:2549-2457

29. Nishio K, Ishida T, Arioka H, Kurokawa H, Fukuoka K (1996) Antitumor effects of butyrolactone I, a selective cdc2 kinase inhibitor, on human lung cancer cell lines. Anticancer Res 16(6B):3387-3395
30. Suzuki M, Hosaka Y, Matsushima H, Goto T, Kitamura T, Kawabe K (1999) Butyrolactone I induces cyclin B1 and causes G2/M arrest and skipping of mitosis in human prostate cell lines. Cancer Lett 138:121-130. doi:10.1016/ S0304-3835(98)00381-4

31. Ojima N, Takahashi I, Ogura K, Shuichi SS (1976) New metabolites from Aspergillus terreus related to the biosynthesis of aspulvinones. Tetrahedron Lett 13:1013-1014

32. Venkatasubbaiah P, Van Dyke CG, Chilton WS (1992) Phytotoxic metabolites of phoma sorghina, a new foliar pathogen of pokeweed. Mycologia 84:715-723. doi:10.2307/3760381.

33. Shao C, Guo Z, Peng H, Peng G, Huang Z (2007) A new isoprenyl phenyl ether compound from mangrove fungus. Chem Nat Comp 43:377-380. doi:10.1007/s10600-007-0142-x.

34. Yamasaki S, Nobusada M, Sasaki T, Shimada A (1999) 6-Methylsalicylic acid, an antifungal substance, produced by an unidentified fungus, No 3. Kyushu Kyoritsu Daigaku Kogakubu Kenkyu Hokoku 23:67-71

35. Sievertsson H, Nilsson JLG (1970) Analgesic properties of methylsalicylic acids. Acta Pharm Suecica 7:289-292

36. Thomas GJ (1984) Herbicidal activity of 6-methylanthranilic acid and analogs. J Agric Food Chem 32:747-749. doi:10.1021/jf00124a011.

37. Kubo I, Muroi H, Kubo A (1994) Naturally occurring antiacne agents. J Nat Prod 57:9-17. doi:10.1021/np50103a002.

38. Nielsen KF, Smedsgaard J (2003) Fungal metabolite screening: database of 474 mycotoxins and fungal metabolites for dereplication by standardised liquid chromatography-UV-mass spectrometry methodology. J Chromatog A 1002:111-136. doi:10.1016/S0021-9673(03)00490-4.

39. Harper JK, Mulgrew AE, Li JY, Barich DH, Strobel GA, Grant DM (2001) Characterization of stereochemistry and molecular conformation using solid-state NMR tensors. J Am Chem Soc 123:9837-9842. doi:10.1021/ ja0109971.

40. Park SH, Kim DS, Kim WG, Ryoo IJ, Lee DH, Huh CH, Youn SW, Yoo ID, Park KC (2004) Terrein: a new melanogenesis inhibitor and its mechanism. Cell Mol Life Sci 61:2878-2885. doi:10.1007/s00018-004-4341-3.

41. Hosoe T, Moriyama H, Wakana D, Itabashi T, Kawai K (2009) Inhibitory effects of dihydroterrein and terrein isolated from Aspergillus novofumigatus on platelet aggregation. Mycotoxins 59:75-82. doi:10.2520/myco.59.75.

42. Kim DS, Lee HK, Park SH, Lee S, Ryoo IJ (2008) Terrein inhibits keratinocyte proliferation via ERK inactivation and G2/M cell cycle arrest. Exp Dermatol 17:312-317. doi:10.1111/j.1600-0625.2007.00646.x.

43. Macedo JFC, Porto ALM, Marzaioli AJ (2004) Terreinol: a novel metabolite from Aspergillus terreus: structure and C labeling. Tetrahedron Lett 45:53-55. doi:10.1016/j.tetlet.2003.10.128.

44. Lin T, Lu C, She Y (2009) Secondary metabolites of Aspergillus sp. F1, a commensal fungal strain of Trewia nudiflora. Nat Prod Res 23:77-85

45. Haritakun R, Rachtawee $P$, Chanthaket R, Boonyuen N, Isaka M (2010) Butyrolactones from the fungus Aspergillus terreus BCC 4651. Chem Pharm Bull 58:1545-1548. doi:10.1248/cpb.58.1545.

46. Ojima N, Takenaka S, Seto S (1975) Structures of pulvinone derivatives from Aspergillus terreus. Phytochemistry 14:573-576. doi:10.1016/0031-9422(75) 85131-4.

47. Burkholder P, Burkholder IM, Almodovar LR (1960) Antibiotic activity of some marine algae of Puerto Rico. Botanica Marina 2:149-156. doi:10.1515/ botm.1960.2.1-2.149.

48. Sajid I, Fondja YCB, Shaaban KA, Hasnain S, Laatsch H (2009) Antifungal and antibacterial activities of indigenous Streptomyces isolates from saline farmlands: prescreening, ribotyping and metabolic diversity. World J Microbiol Biotechnol 25:601-610. doi:10.1007/s11274-008-9928-7.

doi:10.1186/2191-2858-2-9

Cite this article as: Nagia et al.: Four butyrolactones and diverse bioactive secondary metabolites from terrestrial Aspergillus flavipes MM2: isolation and structure determination. Organic and Medicinal Chemistry Letters 2012 2:9. 